\title{
A capnodynamic method delivers continuous hemodynamic monitoring during abdominal surgery
}

Thorir Svavar Sigmundsson ${ }^{1,2}$, Tomas Öhman ${ }^{1,2}$, Magnus Hallbäck ${ }^{3}$, Anders Oldner ${ }^{1,2}$, Caroline HällsjöSander ${ }^{1,2}$, Håkan Björne ${ }^{1,2}$

${ }^{1}$ Department of Perioperative Medicine and Intensive Care, Karolinska University Hospital, Solna, Sweden, ${ }^{2}$ Department of Physiology and Pharmacology, Karolinska Institutet, Stockholm, Sweden, ${ }^{3}$ Maquet Critical Care AB, Solna, Sweden,

\section{Introduction}

A capnodynamic method continuously calculates non-shunted pulmonary blood flow $\left(\mathrm{CO}_{\mathrm{EPBF}}\right)$ using an automatic expiratory hold in three out of nine breaths during mechanical ventilation. In a porcine model, the method has performed well during different respiratory and circulatory challenges ${ }^{1}$, although as expected, transiently affected during swift changes in mixed venous $\mathrm{CO}_{2}$ and at high shunt levels ${ }^{2}$. Concordance during large $\mathrm{CO}$ changes has been preserved throughout the animal studies. The aim of the current study was to evaluate $\mathrm{CO}_{\mathrm{EPBF}}$ clinically for the first time during abdominal surgery.

\section{Methods}

Effective pulmonary blood flow was calculated and compared to trans-pulmonary thermodilution (TPTD) in 25 patients undergoing elective open abdominal surgery. Three measurements were performed at baseline ( $\left(B L_{\text {PEEP5 }}\right)$ at five minutes interval and subsequently before and after changes in PEEP (5-15-5 $\left.\mathrm{cmH}_{2} \mathrm{O}\right)$ and epidural activation with bolus mepivacaine. Events such as hypovolemia and low cardiac output were captured during surgery and values before and after installation of treatment were recorded.

\section{Results}

Mean CO was 4.4 (1.0) and 4.8 (1.1) L/min for EPBF and TPTD in patients with a mean age 68 (9) years and weight 76 (16) kg (see figure 1 for eventline). Bias (levels of agreement) for all 233 paired values was $-0.3 \mathrm{~L} / \mathrm{min}$ and improved after $\triangle$ PEEP 15 without affecting LoA (-2.1 to $1.4 \mathrm{~L} / \mathrm{min})$ and percentage error (37\%) (See figure 2). Concordance with a 15\% exclusion zone $(0.75 \mathrm{~L} / \mathrm{min})$ was 81 and $95 \%$ after PEEP increase and decrease respectively, $86 \%$ after epidural activation and 100\% after volume infusion (See figure 3).

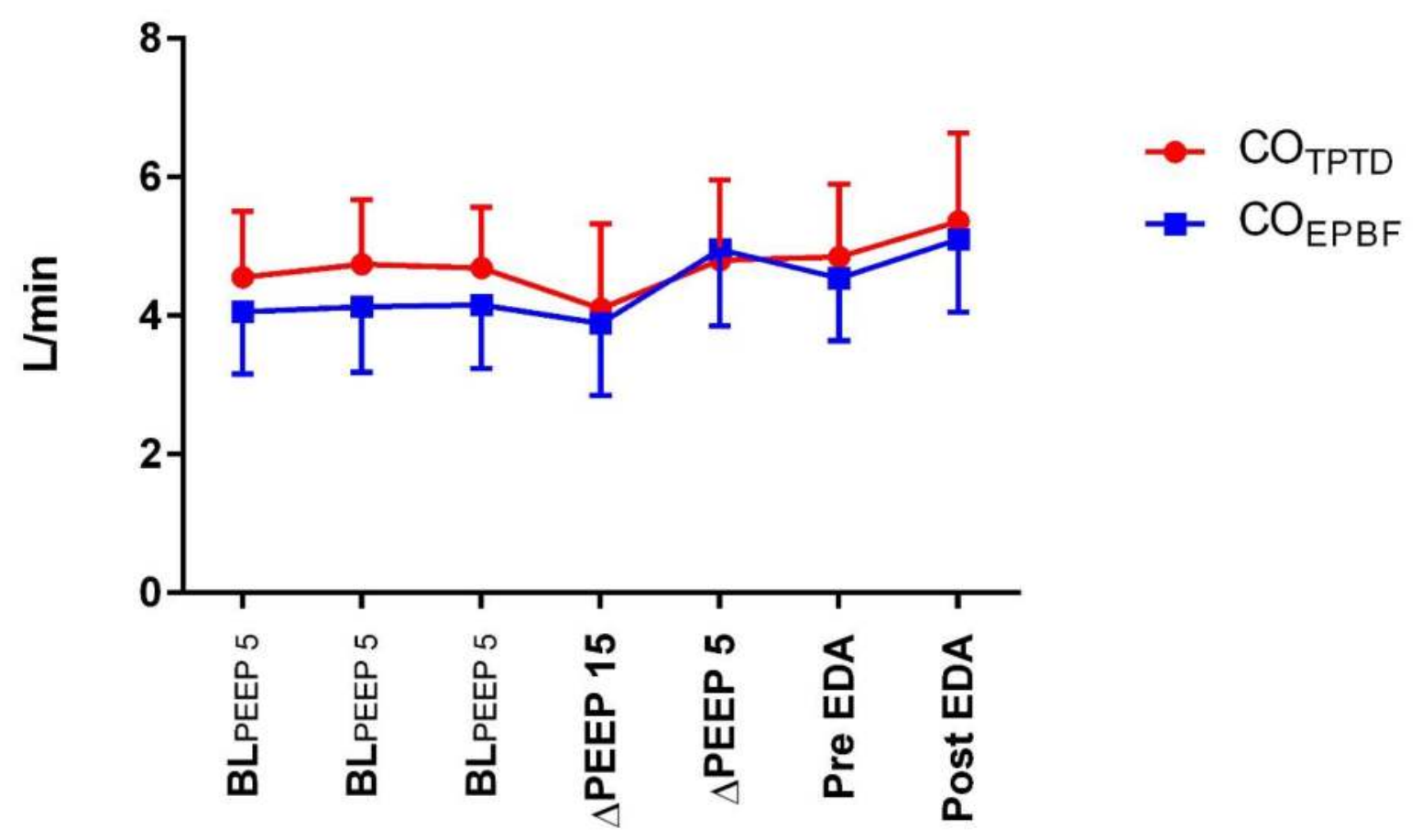

Figure 1. Eventline showing CO measured with EPBF and TPTD during consecutive steps in the protocol

\section{Conclusion}

$\mathrm{CO}_{\text {EPBF }}$ performed acceptably during open abdominal surgery tracking small but recognizable hemodynamic changes starting at PEEP 5. Further clinical studies are warranted to establish its role as a continuous and simple alternative for monitoring and tracking changes in $\mathrm{CO}$ in the operating theatre.

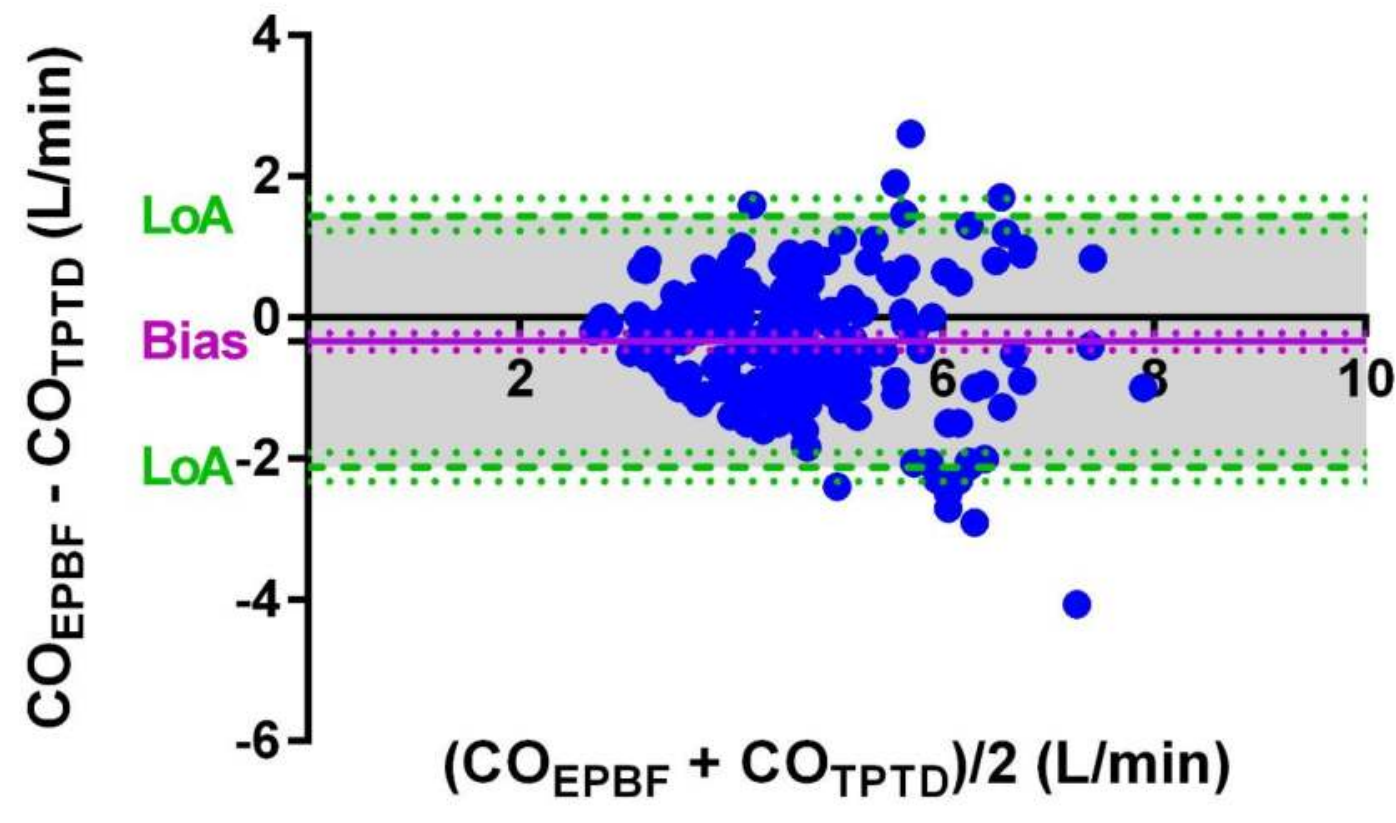

Figure 2. Bland Altman plot for all 233 values

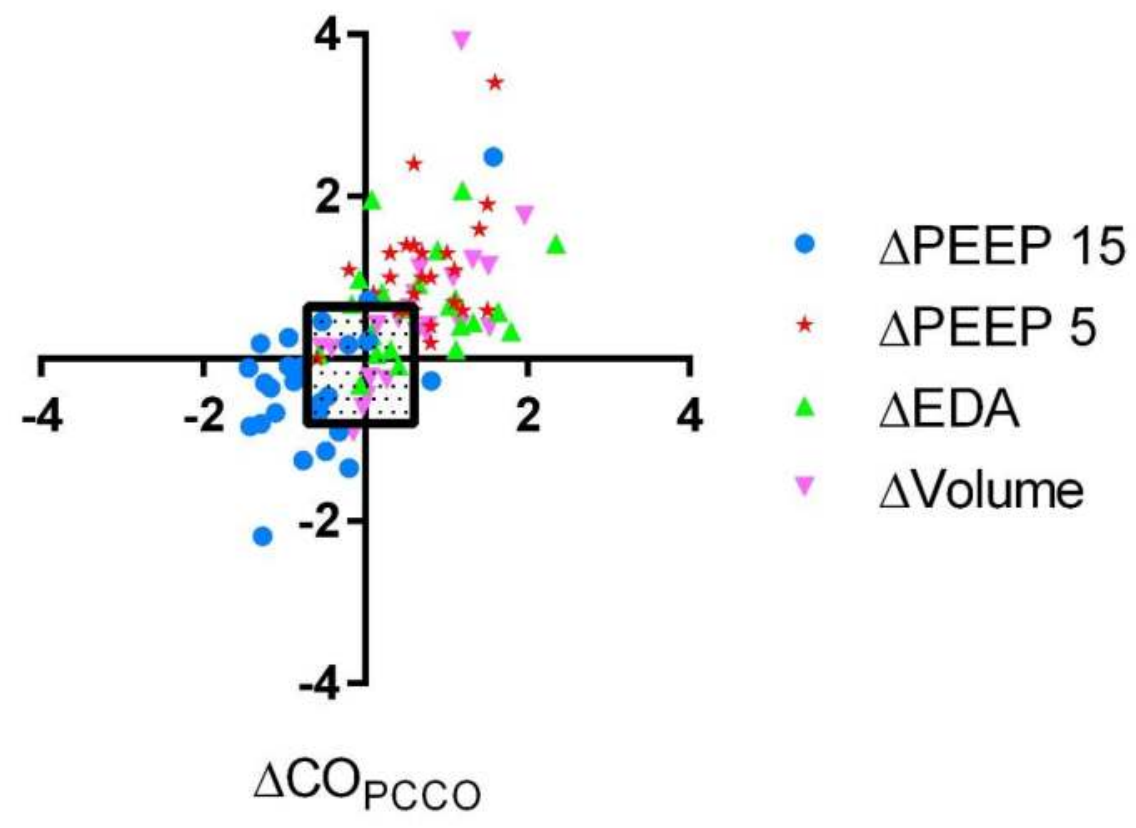

Figure 3. Concordance plot for all trending values with $15 \%$ exclusion zone 